\title{
Pc3 pulsations during variable IMF conditions
}

\author{
U. Villante ${ }^{1}$, S. Lepidi ${ }^{1,2}$, P. Francia ${ }^{1}$, M. Vellante ${ }^{1}$, A. Meloni ${ }^{2}$, R. P. Lepping ${ }^{3}$, F. Mariani ${ }^{4}$ \\ ${ }^{1}$ Dipartimento di Fisica, Università dell'Aquila, L'Aquila, Italy \\ 2 Istituto Nazionale di Geofisica, Roma, Italy \\ 3 Laboratory for Extraterrestrial Physics, NASA/GSFC, Greenbelt, USA \\ 4 Dipartimento di Fisica, Universita' di Roma-Tor Vergata, Italy
}

Received: 11 March 1998 / Revised 26 August 1998 / Accepted: 17 September 1998

\begin{abstract}
Pc3 geomagnetic field fluctuations detected at low latitude (L'Aquila, Italy) during the passage of a high velocity solar wind stream, characterized by variable interplanetary magnetic field conditions, are analyzed. Higher frequency resonant fluctuations and lower frequency phenomena are simultaneously observed; the intermittent appearance and the variable frequency of the longer period modes can be well interpreted in terms of the variable IMF elements; moreover their polarization characteristics are consistent with an origin related to external waves propagating in antisunward direction. A comparison with simultaneous observations performed at Terra Nova Bay (Antarctica) provides additional evidence for a clear relationship between the IMF and Pc3 pulsations also at very high latitudes.
\end{abstract}

Key words. Magnetospheric physics (MHD waves and instabilities; solar wind - magnetosphere interactions)

\section{Introduction}

The physical characteristics of geomagnetic micropulsations and their association with interplanetary and magnetospheric structures are important tools for a better understanding of the primary sources of such phenomena. In this sense, an empirical relationship between the dominant frequency of the dayside Pc3 pulsations $(20 \mathrm{mHz}<f<100 \mathrm{mHz})$ and the strength, $\mathrm{B}$, of the interplanetary magnetic field (hereafter IMF) has been found in several investigations $[\mathrm{f}(\mathrm{mHz}) \sim 6 \mathrm{~B}$ (nT), review by Troitskaya and Bolshakova, 1988]. This relationship has been interpreted as an important

Correspondence to: U. Villante argument in favor of the model predicting that these pulsations could be directly related to ion cyclotron upstream waves generated in the foreshock region along IMF lines connected to the quasi-parallel bow shock (i.e. where the angle between the IMF and the bow shock normal, $\theta_{\mathrm{BN}}$, is less than $45^{\circ}$, Yumoto, 1986). As a consequence, given the average spiral orientation of the IMF lines, these Pc3 pulsations should be preferentially expected in the morning side of the magnetosphere. Moreover small values of the cone angle (the angle $\theta_{\mathrm{XB}}$ between the IMF and the Sun-Earth direction) have been shown to provide favorable conditions for a better transmission of upstream phenomena into the Earth's magnetosphere (Greenstadt et al., 1980; Russell et al., 1983; Odera, 1986). According to Russell et al. (1983), the cone angle control of the ground activity might be dependent on latitude of the observing station and more efficient at lower latitudes. They conducted, indeed, a statistical analysis of the experimental observations between $L=2.4$ and $L=4.3$, and found that for small cone angles $\left(\theta_{\mathrm{XB}}<15^{\circ}\right)$ the normalized rate of occurrence of Pc3 and Pc4 pulsations increases with decreasing latitudes. Recently, Le and Russell (1996) found that the cone angle can also play a role in determining the frequency of upstream waves, although the IMF strength is the most important parameter that controls this frequency.

Several papers have been so far devoted to the analysis of individual events and short time intervals. In this sense, Webb and Orr (1976) found a distinct correspondence between the enhancements in the radial IMF component and the amplitude of Pc3 pulsations at $2.6<L<3.6$; nevertheless, the correlation coefficient between these two parameters was only of the order of 0.135 over time intervals of $16 \mathrm{~h}$. Greenstadt and Olson (1976) also found, at middle latitudes, a clear tendency for signal enhancement for $\theta_{\mathrm{XB}}<50^{\circ}$, although with an appreciable variability in individual cases. More recently, Chi et al. (1994), who analyzed the geomagnetic field observations from a chain of stations $(2.7<L<6.3)$ during a $12 \mathrm{~h}$ interval in which the cone angle was 
smaller than $45^{\circ}$, confirmed a global control of the wave occurrence by the IMF orientation, in that the ground activity abruptly ceased when the cone angle increased to large angles; nevertheless, within this interval, they also found temporal variations of $\mathrm{Pc} 3$ and Pc4 pulsations which were not associated with corresponding variations of the IMF orientation. At lower latitudes ( $L=1.6)$ Villante et al. (1992) examined three different days in 1985 during which the solar wind velocity remained high and approximately constant and showed that the ground power of Pc3 pulsations was clearly switched by variations of the cone angle.

On the other hand, deep into the magnetosphere, upstream waves are also expected to trigger eigenoscillations of the local field lines (Yumoto and Saito, 1983), if the source spectrum of external fluctuations contains the corresponding resonant frequencies: so, in general, both irregular wave forms (with periods related to the IMF magnitude) and more regular oscillations (with latitude dependent periods) are to be expected in ground observations. A previous statistical analysis in this sense, conducted by Vellante et al. (1996, see also Vellante et al., 1989), demonstrated that during years of minimum solar activity the daytime pulsation activity at L'Aquila (Italy, AQ) is typically characterized by the occurrence of two dominant oscillation frequencies, at $\sim 40 \mathrm{mHz}$ and $\sim 80 \mathrm{mHz}$, respectively interpreted in terms of the dominant upstream frequency and of the local fundamental field line eigenfrequency. The lower frequency peak is typically detected in both components while, in agreement with theory, the resonant peak much more sharply emerges in the $\mathrm{H}$ component and is clearly evidenced by the ratio between the $\mathrm{H}$ and $\mathrm{D}$ spectra. Approaching maximum solar activity, due to the simultaneous increase of the IMF strength and plasmaspheric ion density, the two peaks become progressively intermingled, and a single power enhancement in the Pc3 range is more typically observed at solar maximum.

In the present study we analyze the physical characteristics of individual Pc3 wave packets detected at low latitude on January 11, 1997, during variable IMF conditions. As in the statistical analysis conducted by Vellante et al. (1996), we find the simultaneous occurrence of higher frequency resonant fluctuations and lower frequency phenomena; the intermittent appearance and the variable frequency of the longer period modes can be well interpreted in terms of the variable IMF elements. We also analyzed simultaneous observations performed at an Antarctic station (Terra Nova Bay, TNB) and found that also at high latitudes Pc3 pulsations are clearly related to the IMF parameters (Engebretson et al., 1986, 1991).

\section{Data analysis and experimental observations}

The micropulsation measuring systems at AQ and TNB basically consist of three high-sensitivity search-coil magnetometers. At AQ (IGRF95 geomagnetic latitude $\left.\theta=42.5^{\circ}, \quad \mathrm{LT}=\mathrm{UT}+1, \quad \mathrm{MLT}=\mathrm{UT}+1: 40\right)$ the output voltage signals, originally sampled at $16 \mathrm{~Hz}$, are low-pass filtered and finally stored at a sampling rate of $1 \mathrm{~Hz}$. At TNB $\left(\theta=-77.3^{\circ}, \quad \mathrm{LT}=\mathrm{UT}+13\right.$, MLT $=U T-8)$ the data, originally sampled at $10 \mathrm{~Hz}$, are averaged and stored at $1 \mathrm{~Hz}$; unfortunately, in the period of interest, only measurements of the $\mathrm{D}$ component were available. TNB, which during the major part of the day is located in the polar cap, progressively moves toward the polar cusp approaching local geomagnetic noon. Power spectra of the geomagnetic field components have been evaluated by means of the maximum entropy method.

As discussed by Villante et al. (1998), on January 10, 1997, the Earth's passage of a wide magnetic cloud triggered an intense geomagnetic activity at both the low latitude and the Antarctic station and different solar wind pressure variations found correspondence, after several minutes, in strong power enhancements at low frequencies $(\sim 0.8-5 \mathrm{mHz})$. Following the cloud, a solar wind stream, characterized by a velocity greater that $500 \mathrm{~km} / \mathrm{s}$, was detected from WIND $\left(\sim 85 R_{e}\right.$ in the Earth-Sun direction) at $\sim 0300$ UT on January 11, 1997.

In Fig. 1 we compare for the time interval 0500-2400 UT (January 11, 1997), the 10 min running averages (with a step size of $2 \mathrm{~min}$ ) of the IMF magnitude and direction with the running averages of the Pc3 power at $\mathrm{AQ}(\mathrm{H}+\mathrm{D})$ and at TNB (D component). As can be seen, the IMF strength shows a rapid decrease from $\sim 20$ to $\sim 8 \mathrm{nT}$ until $\sim 0800$ UT followed by minor variations around $\sim 6 \mathrm{nT}$; meanwhile the IMF maintains approximately its spiral orientation $\left(\phi \sim 135^{\circ}\right)$, except for two short intervals $(\sim 0520-0540$ UT, $0740-$ 0815 UT).

Several ground power enhancements are detected at AQ in the morning and noon sector $(\sim 0845-0930$ UT, $\sim 1000-1015$ UT, $\sim 1045-1130$ UT): they are mostly observed to occur within intervals characterized, at WIND position, by small values of the cone angle $\left(\theta_{\mathrm{XB}}<45^{\circ}\right)$. In a single case $(\sim 0700-0730$ UT), moreover, the ground power enhancement is somewhat delayed with respect to the occurrence of IMF variations which provided, for a short interval, minimum $\theta_{\mathrm{XB}}$ values of the order of $55^{\circ}$. Actually, similar values of the cone angle (which are not accompanied by significant ground power enhancements) are also detected approximately between $\sim 0730-0800 \mathrm{UT}$; however, as previously mentioned, they mostly correspond to large values of the $\phi$ angle $\left(>200^{\circ}\right)$ which, at the Earth position, would not provide IMF lines connected with the prenoon bow shock. According to Fig. 1, the Pc3 power enhancements at AQ reach approximately the same level, although associated with cone angle values which, on average, become progressively more favorable from the local dawn to the local noon. This feature suggests that the amplitude of the ground pulsations could also depend on the local time, as a consequence of the increasing longitudinal distance from the region where the upstream wave amplitude is expected to maximize (early morning sector for a spiral IMF orientation). 


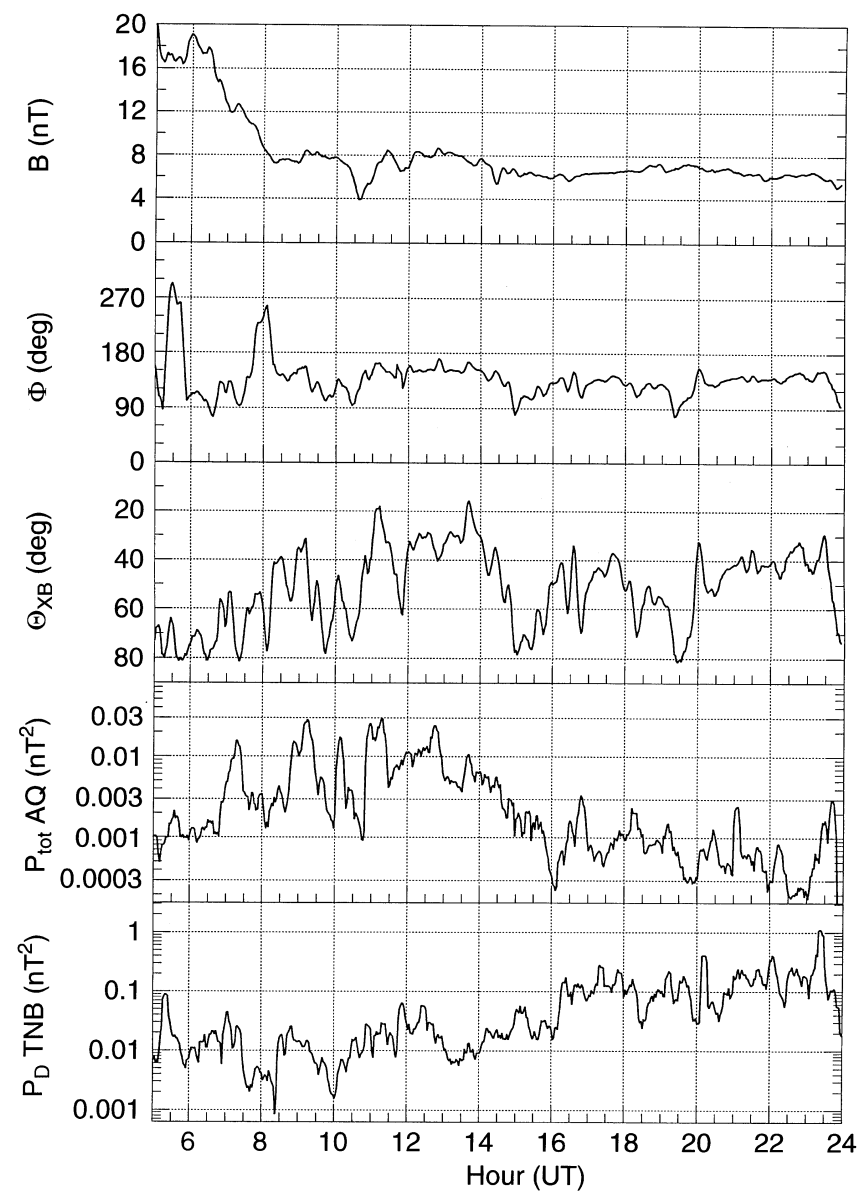

Fig. 1. Interplanetary magnetic field parameters (from the top: magnitude, longitude and cone angle), Pc3 power at AQ $(\mathrm{H}+\mathrm{D})$ and TNB (D component) for the time 0500-2400 UT on January 11, 1997. Powers have been computed by taking into account instrument transfer functions

Consistently, during early afternoon the pulsation activity progressively decays even in presence of favorable cone angle values $(\sim 1200-1430$ UT $)$; it persists, however, some correspondence between minor power enhancements and highly favorable $\theta_{\mathrm{XB}}$ values $(\sim 1340$ UT, $\sim 1645$ UT). During local late afternoon and night the pulsation activity becomes negligible.

The bottom panel in Fig. 1 shows that also at TNB the Pc3 activity is significantly enhanced when the station is located in the dayside magnetic hemisphere (1600-2400 UT, Lepidi et al., 1996). It is interesting to note that also at these high latitudes several power peaks (for example at $\sim 2010$ UT and $\sim 2320$ UT) are observed in correspondence with the smallest cone angle values.

To better analyze the correspondence between the cone angle and the pulsation power, we examined the behavior of the correlation coefficient $r$ between the logarithm of ground Pc3 power and $\cos \left(\theta_{\mathrm{XB}}\right)$ for different delay times: the results of our analysis (Fig. 2) show that the best correspondence is obtained during magnetic daytime hours at both stations for a delay time of the order of $10 \mathrm{~min}(r=0.61$ between 0600 and $1500 \mathrm{UT}$ at AQ; $r=0.60$ between 1500 and
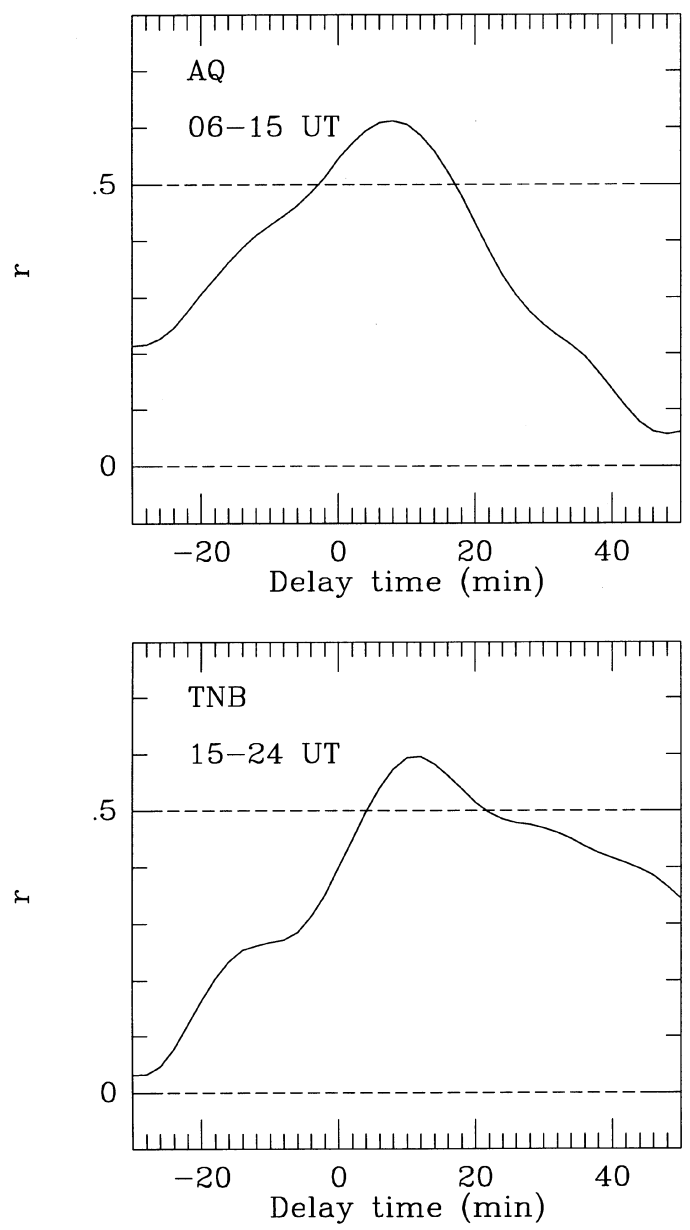

Fig. 2. Normalized cross-correlation function between $\cos \left(\theta_{\mathrm{XB}}\right)$ and the logarithm of the Pc3 power at AQ (upper panel) and TNB (lower panel)

2400 UT at TNB). Moreover, only for TNB, we found that, also during night-time hours, there is some evidence for a dependence of the ground Pc3 power on $\cos \left(\theta_{\mathrm{XB}}\right)$, with approximately the same delay time $(r=0.38$ between 0600 and 1500 UT). The observed delay time $(\sim 10 \mathrm{~min})$ is comparable with the one estimated as the sum of the corotation delay from WIND to the subsolar point of the bow shock ( $\sim 5$ min, assuming the average IMF orientation in the period of interest) and the transmission delay from the bow shock to the ground ( $\sim 6 \mathrm{~min}$; Farrugia et al., 1989).

The dynamic spectra in Fig. 3 clearly confirm the intermittent occurrence of the power enhancements at AQ (upper panels); they also show, more clearly in the $\mathrm{D}$ component, a progressive decrease in frequency (from $\sim 90 \mathrm{mHz}$ to $\sim 40 \mathrm{mHz}$ ) which finds correspondence in the simultaneous decrease of the IMF strength (Fig. 1). In several cases a double peak structure appears in the $\mathrm{H}$ component $(\sim 0720 \mathrm{UT}, \sim 0850$ $0940 \mathrm{UT}, \sim 1010 \mathrm{UT}, \sim 1120 \mathrm{UT})$; this feature can be well interpreted in terms of resonant oscillations of the local field line (Vellante et al., 1989; 1996). An analysis of the behavior of the H/D spectral ratio (Baransky 

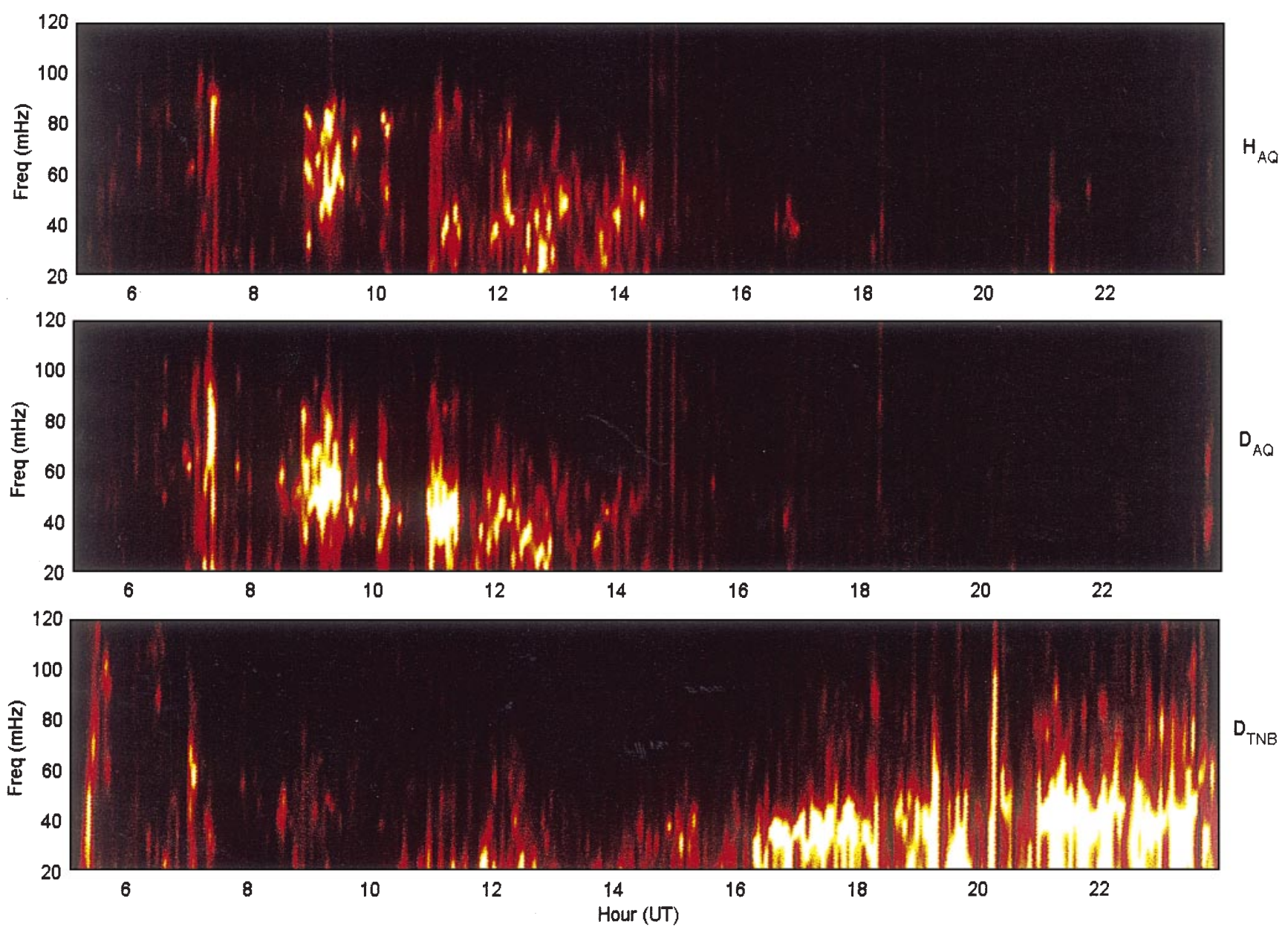

Fig. 3. Dynamic power spectra (obtained by computing power spectra over consecutive 4-min time intervals) of the $\mathrm{H}$ and $\mathrm{D}$ components at $\mathrm{AQ}$ (upper panels) and D component at TNB (lower panel) on January 11, 1997

et al., 1990; Vellante et al., 1993), shows that the resonant frequency is somewhat higher in the early morning ( $~ 90 \mathrm{mHz}$ between 0700 and $0730 \mathrm{UT})$ and then decreases to values of the order of $\sim 80 \mathrm{mHz}$ in the following hours.

As regard TNB, the dynamic spectrum (Fig. 3, lower panel) reveals evidence for enhanced activity in the frequency range $20-60 \mathrm{mHz}$ in the entire dayside magnetic hemisphere, with a slight increase of the dominant frequency until $\sim 2000$ UT, followed by a slight decrease. A similar feature can be observed during the same time interval in the IMF strength (Fig. 1). It is also worth noting some short living narrow-band events during local night-time interval which find correspondence in simultaneous similar features in the AQ spectra $(f \sim 80-90 \mathrm{mHz}$ at $\sim 0630 \mathrm{UT}$ and $f \sim 60 \mathrm{mHz}$ at $\sim 0700$ UT).

In order to better analyze the relationship between the Pc3 frequency and the IMF strength, we visually selected for both stations the clearest wave packets and estimated their frequency from the power spectra of the $\mathrm{D}$ component (which is less affected by resonant phenomena). Figure 4 shows the scatter plots of the selected frequencies versus the corresponding IMF strength (according to previous results, a delay time of 10 min has been assumed). It can be seen that at both stations the correlation between the two parameters is very good ( $r=0.89$ at AQ; $r=0.99$ at TNB); linear regression at a $95 \%$ confidence level gives the empirical relationships: $f(\mathrm{mHz})=(6.2 \pm 0.4) \mathrm{B}(\mathrm{nT})$ and $f$ $(\mathrm{mHz})=(5.7 \pm 0.2) \mathrm{B}(\mathrm{nT})$ at AQ and TNB, respectively. We also investigated a possible influence of the cone angle on the frequency of the observed Pc3 pulsations (Le and Russell, 1996). We found however only a poor correlation $(r=0.35$ at AQ; $r=0.09$ at TNB) between $\mathrm{f} / \mathrm{B}$ and $\cos \left(\theta_{\mathrm{XB}}\right)$.

Finally, a polarization analysis for the selected wave packets at AQ shows highly polarized pulsations with a counterclockwise sense of polarization and polarization axis in the NW-SE direction in the local morning and early afternoon. In Fig. 5 (left side) we show, as an example, the low-pass filtered $(0-100 \mathrm{mHz})$ data in the time interval 0909-0916 UT, together with the corresponding power spectra and hodogram for the signal filtered at the frequency of the main peak $(51 \pm 5 \mathrm{mHz})$. For comparison, we also show in 

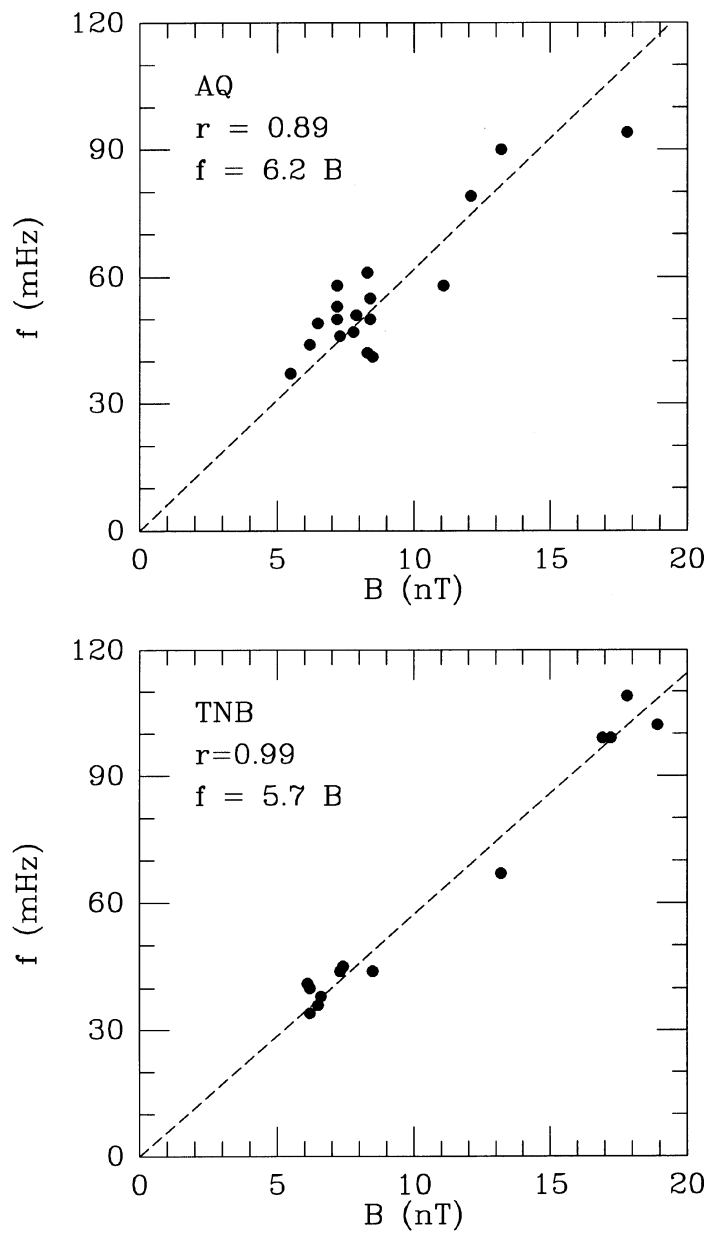

Fig. 4. Dependence of the frequency of selected Pc3 wave packets at $\mathrm{AQ}$ (upper panel) and TNB (lower panel) on the IMF strength. The dashed lines are the linear regressions

Fig. 5 (right side) the short, unique wave packet selected in the local late afternoon (1643-1646 UT); we found in this case a clockwise sense of polarization with a polarization axis switched to NE-SW direction. The observed ellipses orientation and polarization sense can be considered consistent with the predictions drawn for external source waves propagating westward in the morning and eastward in the afternoon hemisphere (Chen and Hasegawa, 1974).

\section{Summary and discussion}

In this study we analyzed the high resolution geomagnetic field measurements at low and high latitude during the Earth's passage of a high velocity solar wind stream characterized by variable IMF conditions.

At low latitudes, during local morning, Pc3 power intensifications were simultaneously observed in both components: the observed decrease of the dominant frequency in a wide range was well consistent with the corresponding decrease of the IMF strength, and the intermittent character of the pulsation activity was found to be related with the variable orientation of the IMF lines. These features both suggest that the ground wave packets can be well interpreted in terms of upstream ion cyclotron waves penetrating deep into the magnetosphere (Yumoto, 1986). Russell et al. (1983) suggested that low latitude ground pulsations are mostly generated close to the subsolar point and propagate nearly radially inwards. Our observations suggest that also upstream waves generated in the morning side of the bow shock (where for nominal IMF conditions the level of turbulence is higher) can reach low latitudes. On the other hand, similar results were also obtained by previous statistical analysis conducted at low latitudes by Yumoto et al. (1988) and Vellante et al. (1989) who found that the Pc3 activity in the D component (less affected by field line resonance, it more strictly reflects the upstream waves) maximizes during morning hours.

Pulsation measurements at TNB (Antarctica) show that Pc3 activity is enhanced when the station is located in the dayside magnetic hemisphere and modulated by the variable orientation of the IMF lines. In some cases, however, we found evidence for night-time Pc3 fluctuations which were simultaneous with similar dayside fluctuations at low latitudes. This result confirms that in the polar cap solar wind controlled pulsations can be observed also during night-time hours (Engebtretson et al., 1989), supporting the idea of an additional high latitude entry of the upstream ULF wave energy (Engebretson et al., 1991). In this sense we found that, in agreement with previous results (review by Troitskaya and Bolshakova, 1988), both at low and high latitude the dominant wave frequency depends on the strength of the IMF according to the relation $\mathrm{f}$ $(\mathrm{mHz}) \sim 6 \mathrm{~B}(\mathrm{nT})$.

At AQ the polarization analysis of the observed Pc3 pulsations shows opposite sense of polarization and different direction of the polarization axis between morning and afternoon observations. These results are in agreement with those provided by previous investigations at low latitudes (Lanzerotti et al., 1981; Yumoto et al., 1985; Ansari and Fraser, 1986), and are consistent with the polarization pattern expected for waves propagating in the antisunward direction (Southwood, 1974; Chen and Hasegawa, 1974).

The observed fluctuations at AQ were typically accompanied by the simultaneous occurrence, at higher frequencies, of power enhancements which are clearly related to resonance phenomena of local geomagnetic field lines. Estimates of the resonant frequency confirm a tendency for higher values in the early morning hours (Green et al., 1993, Waters et al., 1994).

Acknowledgements. Authors thank A. Piancatelli and M. De Lauretis (Università dell'Aquila) for cooperation in data analysis. This research activity at L'Aquila is supported by PNRA and by GIFCO/CNR. Support by ASI is acknowledged by one of us (F. M.).

Topical Editor K.-H. Glassmeier thanks C. L. Waters and J. Vero for their help in evaluating this paper. 

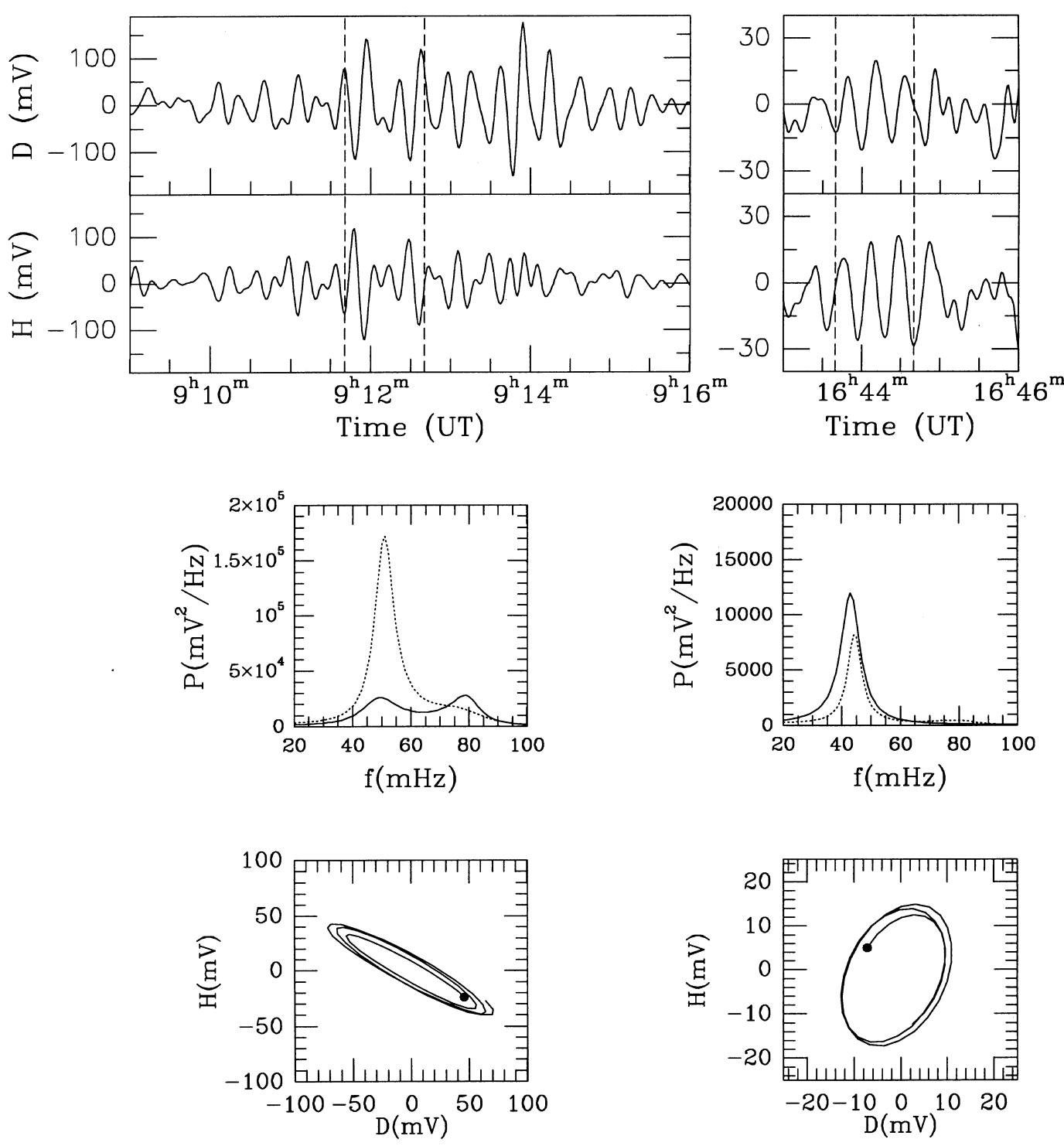

Fig. 5. Low-pass filtered $(0-100 \mathrm{mHz}) \mathrm{H}$ and $\mathrm{D}$ data at $\mathrm{AQ}$ in the time intervals 0909-0916 UT (left) and 1643-1646 UT (right), together with the corresponding power spectra $(\mathrm{H}$, solid line) and the hodograms for two selected wave packets (indicated in the

\section{References}

Ansari, I. A., and B. J. Fraser, A multistation study of low latitude Pc3 geomagnetic pulsations, Planet. Space Sci., 34, 519, 1986.

Baransky, L. N., S. P. Belokris, U. E. Borovkov, and C. A. Green, Two simple methods for the determination of the resonance frequencies of magnetic field lines, Planet. Space Sci., 38, 1573, 1990.

Chen, L., and A. Hasegawa, A theory of long-period magnetic pulsations, 1, steady state excitation of field line resonances, $J$. Geophys. Res., 79, 1024, 1974.

Chi, P. J., C. T. Russell, and G. Le, Pc3 and Pc4 activity during a long period of low interplanetary magnetic field cone angle as detected across the Institute of Geological Sciences array, $J$. Geophys. Res., 99, 11127, 1994.

Engebretson M. J., C.-I. Meng, R. L. Arnoldy, and L. J. Cahill, Jr., Pc3 pulsations observed near the south polar cusp, J. Geophys. Res., 91, 8909, 1986. corresponding upper panel by dashed vertical lines) filtered at the peak frequency $(51 \pm 5 \mathrm{mHz}$ and $44 \pm 5 \mathrm{mHz})$; the black dot indicates the initial point

Engebretson M. J., B. J. Anderson, L. J. Cahill, Jr., R. L. Arnoldy, P. T. Newell, C.-I. Meng, L. J. Zanetti, and T. A. Potemra, A multipoint case study of high latitude daytime ULF pulsations, J. Geophys. Res., 94, 17 143, 1989.

Engebretson M. J., L. J. Cahill, Jr., R. L. Arnoldy, B. J. Anderson, T. J. Rosenberg, D. L. Carpenter, U. S. Inan, and R. H. Eather, The role of the ionosphere in coupling upstream ULF wave power into the dayside magnetosphere, J. Geophys. Res., 96, $1527,1991$.

Farrugia, C. J., M. P. Freeman, S. W. H. Cowley, D. J. Southwood, M. Lockwood, and A. Etemadi, Pressure driven magnetopause motions and attendant response on the ground, Planet. Space Sci., 37, 589, 1989.

Green, A. W., E. W. Worthington, L. N. Baransky, E. N. Fedorov, N. A. Kurneva, V. A. Pilipenko, D. N. Shvetzov, A. A. Bektemirov, and G. V. Philipov, Alfvén field line resonances at low-latitudes (L = 1.5), J. Geophys. Res., 98, 15 693, 1993.

Greenstadt, E. W., and J. V. Olson, Pc3,4 activity and interplanetary field orientation, J. Geophys. Res., 81, 5911, 1976. 
Greenstadt, E. W., R. L. McPherron, and K. Takahashi, Solar wind control of daytime, midperiod geomagnetic pulsations, $J$. Geomagn Geoelectr., 32 (11), 89, 1980.

Lanzerotti, L. J., L. V. Medford, C. G. Maclennan, T. Hasegawa, M. H. Acuna, and S. R. Dolce, Polarization characteristics of hydromagnetic waves at low geomagnetic latitudes, J. Geophys. Res., 86, 5500, 1981.

Le, G., and C. T. Russell, Solar wind control of upstream wave frequency, J. Geophys. Res., 101, 2571, 1996.

Lepidi, S., U. Villante, M. Vellante, P. Palangio, and A. Meloni, High resolution geomagnetic field observations at Terra Nova Bay, Antarctica, Ann. Geofis., 34, 519, 1996.

Odera, T. J., Solar wind controlled pulsations: a review, Rev. Geophys., 24, 55, 1986.

Russell, C. T., J. G. Luhmann, T. J. Odera, and W. F. Stuart, The rate of occurrence of dayside Pc3,4 pulsations: the L-value dependence of the IMF cone angle effect, J. Geophys. Res., 10, 663,1983

Southwood, D. J., Some features of field-line resonances in the magnetosphere, Planet. Space Sci., 22, 483, 1974.

Troitskaya, V. A., and O. V. Bolshakova, Diagnostics of the magnetosphere using multipoint measurements of ULF-waves, Adv. Space Res., 8, 413, 1988.

Vellante, M., U. Villante, M. De Lauretis, and P. Cerulli-Irelli, An analysis of micropulsation events at a low-latitude station during 1985, Planet. Space Sci., 37, 767, 1989.

Vellante, M., U. Villante, R. Core, A. Best, D. Lenners, and V. A. Pilipenko, Simultaneous geomagnetic pulsation observations at two latitudes: resonant mode characteristics, Ann. Geophysicae, 11, 734, 1993.
Vellante, M., U. Villante, M. De Lauretis, G. Barchi, Solar cycle variation of the dominant frequencies of Pc3 geomagnetic pulsations at L = 1.6, Geophys. Res. Lett., 23, 1505, 1996.

Villante, U., S. Lepidi, M. Vellante, A. J. Lazarus, and R. P. Lepping, Pc3 activity at low geomagnetic latitudes: a comparison with solar wind observations, Planet. Space Sci., 40, 1399, 1992.

Villante, U., M. De Lauretis, P. Francia, S. Lepidi, L. Cafarella, A. Meloni, A. J. Lazarus, R. P. Lepping, and F. Mariani, Low frequency geomagnetic field variations at low and high latitude during the January 10-11, 1997 magnetic cloud, Geophys. Res. Lett., 25, 2593, 1998.

Waters, C. L., F. W. Menk, and B. J. Fraser, Low latitude geomagnetic field line resonance: experiment and modeling, $J$. Geophys. Res., 99, 17 547, 1994.

Webb, D., and D. Orr, Geomagnetic pulsations $(5-50 \mathrm{mHz})$ and the interplanetary magnetic field, J. Geophys. Res., 81, 5941, 1976.

Yumoto, K., Generation and propagation mechanisms of lowlatitude magnetic pulsations - a review, J. Geophys., 60, 79, 1986.

Yumoto, K., and T. Saito, Relation of compressional HM waves at GOES 2 to low-latitude Pc3 magnetic pulsations, J. Geophys. Res., 88, 10 041, 1983.

Yumoto, K., T. Saito, and Y. Tanaka, Low-latitude Pc3 magnetic pulsations observed at conjugate stations $(\mathrm{L} \sim 1.5), J$. Geophys. Res., 90, 12 201, 1985.

Yumoto, K., T. Saito, Y. Tanaka, K. J. W. Lynn, F. W. Menk, and B. J. Fraser, Polarization and amplitude characteristics of Pc3 pulsations in conjugate area around $\mathrm{L}=1.3-2.1, \mathrm{~J}$. Geophys. Res., 93, 7386, 1988. 